\title{
RAYNAUD'S GROUP-SCHEME AND REDUCTION OF COVERINGS
}

\author{
DAN ABRAMOVICH
}

\section{INTRODUCTION}

1.1. Reduction of coverings of degree $p$. Let $R$ be a discrete valuation ring of mixed characteristics, $S=\operatorname{Spec} R$, with generic point $\eta$ with fraction field $K$ and special point $s$ with residue field $k$ of characteristic $p>0$. Consider a generically smooth, stable marked curve $Y \rightarrow S$ with an action of a finite group $G$ of order divisible by $p$. Denote $X=Y / G$. We assume that $G$ acts freely on the complement of the marked points in $Y_{\eta}$ (it follows that $G$ respects the branches of all nodes of $Y_{s}$ ).

In situations where the order of $G$ is prime to the residue characteristic, the reduced covering $Y_{s} \rightarrow X_{s}$ is an admissible $G$-covering, and a nice complete moduli space of admissible $G$ coverings exists. An extensive literature exists describing that situation. However, in our case where the residue characteristic divides the order of $G$, interesting phenomena occur (see e.g. $\aleph-02$ ]). The situation was studied by a number of people; we will concern ourselves with results of Raynaud $\mathrm{Ra}$ ] and, in a less direct way, Henrio [He]. Related work of Saidi Sa1, Sa2, Sa3, Wewers and Bouw W1, W2, W3, Bo, B-W1, B-W2, Romagny [Ro] and others provides additional inspiration.

Thus, in our case where $p|| G \mid$, the covering $Y \rightarrow X$ is no longer generically étale on each fiber. It is natural to consider some sort of group-scheme degeneration $\mathcal{G} \rightarrow X$ of $G$, in such a way that $Y$ might be considered something like an admissible $\mathcal{G}$-covering. Raynaud ([Ra], Proposition 1.2.1) considered such a degeneration locally at the generic points of the irreducible components of $X_{s}$, in the special case where $|G|=p$; in our first result below we will work out its extension to the smooth locus of $X$ (Theorem 3.1.1), and slightly more general groups, where $p^{2} \nmid|G|$ and the $p$-Sylow subgroup of $G$ is normal. The case where $p^{2}|| G \mid$ remains a question which I find very interesting (see example 2.1.5 for a negative result in general and remark 2.1.6 for a positive result for small ramification).

One still needs to understand the structure of $Y \rightarrow X$ at the nodes of $X_{s}$ and $Y_{s}$. Henrio, working $p$-adic analytically, derived algebraic data along $X_{s}$, involving numerical invariants and differential forms, which in some sense classify $Y_{s} \rightarrow X_{s}$. Our second goal in this note is to present a slightly different approach to such degenerations at a node, modeled on twisted curves, i.e. curves with algebraic stack structures. The point is that, just as in $\aleph-\mathrm{C}-\mathrm{V}$, the introduction of twisted curves allows to replance $Y \rightarrow X$ by something that is more like a principal bundle. Unlike the case of residue characteristics prime to $|G|$, the twisted curves will in general be Artin stacks rather than Deligne-Mumford stacks. Moreover, we will need to add two levels of twistings: one seems to be needed for extending the degenerate group scheme (but see Question 3.2.2); the second makes the map $Y \rightarrow X$ into a principal bundle under the resulting group scheme.

Research partially supported by NSF grant DMS-0070970, a Forscheimer Fellowship and Landau Center Fellowship. 
1.2. Acknowledgements. Thanks to Angelo Vistoli for help, and to F. Andreatta, A. Corti, A.J. de Jong, and N. Shepherd-Barron for patient ears and useful comments. I also heartily thank Jonathan Lubin, who pointed me in the direction of Example 2.1.5, and in particular saved me from desparate efforts to prove results when $p^{2}|| G \mid$.

2. EXtensions of GROUPS SCHEMES AND THEIR ACTIONS IN DIMENSION 1 AND 2

2.1. Raynaud's group scheme. Raynaud (see [Ra], Proposition 1.2.1, see also Romagny, [Ro] ) considers the following construction: let $U$ be integral and let $V / U$ be a finite flat $G$ invariant morphism of schemes, with $G$ finite. Assume that the action of $G$ on the generic fiber of $V / U$ is faithful. We can view this as an action of the constant group scheme $G_{U}$ on $V$, and we consider the schematic image $\mathcal{G}$ of the associated homomorphism of group schemes

$$
G_{U} \rightarrow \operatorname{Aut}_{U} V \text {. }
$$

Since, by definition, $G_{U} \rightarrow U$ is finite, we have that $\mathcal{G} \rightarrow U$ is finite as well. The scheme $\mathcal{G} \rightarrow U$ can also be recovered as the closure of the image of the generic fiber of $G_{U}$, which is, by the faithfulness assumption, a subscheme of $\operatorname{Aut}_{U} V$. By definition $\mathcal{G}$ acts faithfully on $V$.

Definition 2.1.1. We call the scheme $\mathcal{G}$ the effective model of $G$ acting on $V / U$.

Note that a-priori we do not know that $\mathcal{G}$ is a group-scheme. It is however automatically a flat group scheme if $U$ is the spectrum of a Dedekind domain. (This follows because, in that case, the image of $\mathcal{G} \times{ }_{U} \mathcal{G} \rightarrow \operatorname{Aut}_{U} V$ is also flat, and therefore must coincide with $\mathcal{G}$ ).

Also note that, if $s$ is a closed point of $U$ whose residue characteristic is prime to the order of $G$, then the fiber of $\mathcal{G}$ over $s$ is simply $G$. So this faithful model is only of interest when the residue characteristic divides $|G|$.

I recall and slightly extend a result of Raynaud (see [Ra], Proposition 1.2.1):

Proposition 2.1.2. Let $U$ be the spectrum of a discrete valuation ring, with special point $s$ of residue characteristic $p$ and generic point $\eta$. Let $V \rightarrow U$ be a finite and flat morphism, and assume that the fiber $V_{s}$ of $V$ over $s$ is reduced (but not assuming geometrically reduced). Assume given a finite group $G$, with normal $p$-Sylow subgroup, such that $p^{2} \nmid|G|$, and an action of $G$ on $V$ such that $V \rightarrow U$ is $G$-invariant, and such that the generic fiber $V_{\eta} \rightarrow\{\eta\}$ is a principal homogeneous space. Let $\mathcal{G} \rightarrow U$ be the effective model of $G$ acting on $V / U$.

Then $V / U$ is a principal bundle under the action of $\mathcal{G} \rightarrow U$.

Proof. As in Raynaud's argument, it suffices to show that the stabilizer of the diagonal of $V_{s} \times_{U} V_{s}$ inside the group scheme $V_{s} \times_{U} \mathcal{G}$ is trivial. Since $G$ acts transitively on the closed points $t_{i}$ of $V_{s}$ sending the stabilizer on $t_{i}$ to that over $t_{j}$, it is enough to show that one of these stabilizers, say over $t \in V_{s}$, is trivial. But this stabilizer $P$ is a group scheme over a field with $\operatorname{deg} P \mid p$, and if nontrivial it is or degree exactly $p$. In such a case it must coincide with the pullback of the unique $p$-Sylow group-subscheme of $\mathcal{G}$, therefore that $p$-sylow acts trivially, contradicting the fact that $\mathcal{G}$ acts effectively.

Remark 2.1.3. In case the inertia group is not normal, Raynaud passes to an auxiliary cover, which encodes much of the behavior of $V \rightarrow U$.

Question 2.1.4. What can one say on the action of $\mathcal{G}$ on $V$ in case the order of $G$ (and the degree of $V \rightarrow U)$ is divisible by $p^{2}$, but the inertia group is still normal? For instance, does a result similar to Proposition 2.1.2 hold if $|G|=p^{2}$ ?

In the latter case, consider a subgroup $P \subset G$ of order $p$. It can be argued as in Raynaud's proof, that the effective model $\mathcal{P} \rightarrow U$ of $P$ acts freely on $V$, and thus $V \rightarrow V / P$ is a principal 
$\mathcal{P}$-bundle. Similarly, if $\mathcal{Q}$ is the effective model of $G / P$ acting on $V / P$, then $V / P \rightarrow U$ is a principal $\mathcal{Q}$-bundle. At the same time, we have an action of the effective model $\mathcal{G}$ of $G$ on $V / P$, but it is not necessarily the case that $\mathcal{G} / \mathcal{P} \rightarrow \mathcal{Q}$ should be an isomorphism!

Example 2.1.5. As an example, consider the action of $\mathcal{G}_{0}=\left(\alpha_{p}\right)^{2}=\operatorname{Spec} k[a, b] /\left(a^{p}, b^{p}\right)$ on $k(t)$ given by

$$
t \mapsto t+a+t^{p} b
$$

This action is effective but not free.

I claim that at least in characteristic 2, this action can be obtained as a reduction of an action of $(\mathbb{Z} / 2 \mathbb{Z})^{2}$ on a nodal curve. I concentrate on the local picture (making it global is not difficult):

Let $R=\mathbb{Z}_{2}[\sqrt{2}]$. Consider the group-scheme $Y / R$ defined by

$$
t * t^{\prime}=t+t^{\prime}+\sqrt{2} t t^{\prime}
$$

This is an additive reduction of the multiplicative group. The reduction of the subgroup $\mu_{2}$ is given as

$$
\text { Spec } R[a] /(a(a+\sqrt{2})),
$$

reducing to $\alpha_{2}$. It acts on $Y$ by translation via the addition law as above:

$$
t \mapsto t+a+\sqrt{2} a t
$$

The reduction of the action of $\mathbb{Z} / 2 \mathbb{Z}$ by inversion is the same group scheme, again reducing to $\alpha_{2}$, which we write as

$$
\text { Spec } R[b] /(b(b+\sqrt{2})) \text {. }
$$

This time the action is given by

$$
t \mapsto(1+\sqrt{2} b) t-\frac{b t^{2}}{1+\sqrt{2} t}
$$

Since 2-torsion is fixed by inversion, these actions commute. Explicitly, the action of the product is given by

$$
t \mapsto a+(1+\sqrt{2} b) t-\frac{b t^{2}}{1+\sqrt{2} t}+\sqrt{2} a\left((1+\sqrt{2} b) t-\frac{b t^{2}}{1+\sqrt{2} t}\right) .
$$

The reduction modulo $\sqrt{2}$ is given by

$$
t \mapsto t+a+t^{2} b
$$

as required.

It would be interesting to write down an example with odd residue characteristics.

Remark 2.1.6. The arguments do work when $p^{2}|| G \mid$ if the $p$-Sylow group-scheme of $\mathcal{G}$ has only étale and cyclotomic Jordan-Hölder factors. This is because, in that case, the are no nonconstant group subschemes in the reduction. In particular this works whenever the absolute ramification index over $\mathbb{Z}_{p}$ is $<p$. 
2.2. Extension from dimension 1 to dimension 2. Consider now the case where $U$ is a regular scheme with $\operatorname{dim} U=2$. Consider the S2-saturation $\mathcal{G}^{\prime} \rightarrow \mathcal{G}$ of the effective model $\mathcal{G}$ of the $G$ action on $V / U$. We have

Lemma 2.2.1. The S2-saturation $\mathcal{G}^{\prime}$ of the effective model $\mathcal{G}$ is a finite flat group scheme acting on $V$.

Proof. Since $\mathcal{G}^{\prime}$ is S2 and 2-dimensional, it is Cohen-Macaulay, and being finite over the nonsingular scheme $U$, it is finite and flat over $U$ (indeed its structure sheaf, being saturated, is locally free over the nonsingular 2-diemnsional scheme $U$ ). Pulling back to $\mathcal{G}^{\prime}$ the CohenMacaulay flat morphism $\mathcal{G}^{\prime} \rightarrow U$ we have that $\mathcal{G}^{\prime} \times \mathcal{G}^{\prime}$ is $\mathrm{S} 2$ and flat over $\mathcal{G}^{\prime}$ and over $U$.

We claim that the rational map $\mathcal{G}^{\prime} \times_{U} \mathcal{G}^{\prime} \rightarrow \mathcal{G}^{\prime}$ induced by multiplication in $A u t_{U} V$ is everywhere defined. Indeed the graph of this map is finite over $\mathcal{G}^{\prime} \times_{U} \mathcal{G}^{\prime}$ and isomorphic to it over the locus where $\mathcal{G}^{\prime} \rightarrow \mathcal{G}$ is an isomorphism, whose complement has codimension $\geq 2$. The S2 property implies that the graph is isomorphic to $\mathcal{G}^{\prime} \times_{U} \mathcal{G}^{\prime}$, and the map is regular. The same works for the map defined by the inverse in $A u t_{U} V$. This makes $\mathcal{G}^{\prime}$ a group-scheme, and the map $\mathcal{G}^{\prime} \rightarrow A u t_{U} V$ into a group-homomorphism.

When the action on the generic fiber is free, we have more:

Proposition 2.2.2. Let $U$ be a nonsingular integral scheme with $\operatorname{dim} U=2$. Let $V \rightarrow U$ be finite and Cohen-Macaulay, $G$ invariant, and assume the action of $G$ on the generic fiber is free. Let $\mathcal{G} \rightarrow U$ be the effective model of the action. Assume that for every codimension-1 point $\xi$, the action of the fiber $\mathcal{G}_{\xi}$ on $V_{\xi}$ is free.

Then

(1) $\mathcal{G} \rightarrow U$ is a flat group-scheme, and

(2) The action of $\mathcal{G}$ on $V$ is free.

Note that, by Raynaud's proposition, the assumptions hold when $U=V / G$, is local of mixed characteristics $(0, p)$, the fibers $V_{\xi}$ are reduced, the $p$-Sylow of $G$ is normal and $p^{2} \nmid|G|$.

Proof. We have that $\mathcal{G}^{\prime} \times_{U} V \rightarrow V$ is flat with Cohen-Macaulay fibers. The same is true for $V \rightarrow U$. This implies that $\mathcal{G}^{\prime} \times_{U} V \rightarrow U$ is flat and $\mathcal{G}^{\prime} \times_{U} V$ is S2. Also $V \times_{U} V$ is $\mathrm{S} 2$, being flat over $X$. Now by assumption the action of $\mathcal{G}^{\prime}$ on $V$ is free in codimension 1 over $U$. We have a morphism of S2 schemes of dimension 2

$$
\mathcal{G}^{\prime} \times_{U} V \rightarrow V \times_{U} V
$$

which is an isomorphism in codimension 1 , so it is an isomorphism. This implies that $\mathcal{G}^{\prime}$ is a subscheme of $\operatorname{Aut}_{U} V$, and therefore $\mathcal{G}^{\prime}=\mathcal{G}$. The proposition follows.

\section{Curves}

3.1. The smooth locus. The main case of interest for us is the following:

Let $R$ be a complete discrete valuation ring of mixed characteristic, with fraction field $K$ of characteristic 0 , residue field $k$ of characteristic $p>0$, and spectrum $S$. Assume $Y \rightarrow S$ is a stable curve with smooth generic fiber, $G$ a finite group acting on $Y$ over $S$, and denote

$$
X=Y / G \text {. }
$$

We assume that the closure of fixed points on $Y_{K}$ are disjoint integral points of the smooth locus $Y_{\mathrm{sm}}$ and hence for every node $y \in Y$, the stabilizer in $G$ of $y$ keeps the branches of $Y$ at $y$ invariant. We denote the complement of the closure of generic branch points in $Y_{\mathrm{sm}}$ by $Y_{\text {gen }}$, and the image in $X$ by $X_{\text {gen }}$ - the so called general locus. 
Note that $Y_{\mathrm{sm}} \rightarrow X_{\mathrm{sm}}$ is flat.

The propositions above give:

Theorem 3.1.1. Assume $p^{2} \nmid|G|$ and the p-Sylow subgroup of $G$ is normal.

There exist

(1) a finite flat group scheme $\mathcal{G} \rightarrow X_{\mathrm{sm}}$,

(2) a homomorphism $G_{X_{\mathrm{sm}}} \rightarrow \mathcal{G}$ which is an isomorphism on $X_{K}$, and

(3) an action of $\mathcal{G}$ on $Y_{\mathrm{sm}}$ through which the action of $G$ factors,

such that $Y_{\text {gen }} \rightarrow X_{\text {gen }}$ is a principal $\mathcal{G}$-bundle.

The formation of $\mathcal{G}$ commutes with any flat and quasi-finite base change $R \subset R^{\prime}$.

It would be really interesting to see what happens for other groups $G$.

3.2. Flat extension of group scheme over $X$. What can be done about the singular points of $X$ and $Y$ ? It is easy to see that even in the case of characteristic 0 , the cover $Y \rightarrow X$ is not a principal bundle in general (and certainly not at the closure of generic branch points). However, the behavior of $Y \rightarrow X$ at the nodes is very interesting. Several authors, notably Henrio $[\mathrm{He}$, have attached invariants to such a node which say much about the cover $Y \rightarrow X$. It seems desirable to attach a local geometric structure which underlies such invariants. My suggested approach here is to follow the method of [\$-V2, $\aleph-\mathrm{V} 1, \aleph-\mathrm{C}-\mathrm{V}$ ] using twisted curves.

First we need to somehow extend $\mathcal{G}$ over nodes.

Consider a node where $X$ is described locally by $x y-\pi^{r}$, with $\pi$ a uniformizer in $S$ (splitting the nodes is obtained after a quadratic base change and passing to a completion on $X$ ). The $\boldsymbol{\mu}_{r}$-cover

$$
Z=\operatorname{Spec} R[u, v] /(u v-\pi)
$$

given by $x=u^{r}, y=v^{r}$ is regular. Now $Y \times_{X} Z$ is Cohen-Macaulay in codimension 1. Its (partial) normalization $\tilde{Y}_{Z}$ in the structure sheaf of $Y_{\text {gen }} \times_{X} Z$ is S2, so it is Cohen-Macaulay. It follows that $\tilde{Y}_{Z} \rightarrow Z$ is flat. We have a $\boldsymbol{\mu}_{r}$ equivariant action of $G$ on $\tilde{Y}_{Z}$. Taking the S2-saturation of the effective model of this action leads as above to a flat group-scheme $\mathcal{G}_{Z}^{\prime}$ extending $\mathcal{G}_{\text {gen }}$, with an action of $\boldsymbol{\mu}_{r}$ on the morphism $\mathcal{G}_{Z}^{\prime} \rightarrow Z$.

Let $\boldsymbol{\mu}_{r_{0}}$ be the subgroup of $\boldsymbol{\mu}_{r}$ acting trivially on the closed fiber of $\mathcal{G}_{Z}^{\prime}$ at $u=v=0$, and let $r_{1}=r / r_{0}$. Consider the quotient $Z_{1}=Z / \boldsymbol{\mu}_{r_{0}}$. It can be described as

$$
Z_{1}=\operatorname{Spec} R\left[u_{1}, v_{1}\right] /\left(u_{1} v_{1}-\pi^{r_{1}}\right),
$$

with $x=u_{1}^{r_{1}}, y=v_{1}^{r_{1}}$. It comes with an action of $\boldsymbol{\mu}_{r_{1}}$. Since $\boldsymbol{\mu}_{r_{0}}$ is diagonalizable, acting trivially on the fiber of $\mathcal{G}_{Z}^{\prime}$, the quotient

$$
\mathcal{G}_{Z_{1}}^{\prime}=\mathcal{G}_{Z}^{\prime} / \boldsymbol{\mu}_{r_{0}}
$$

is a flat group-scheme over $Z_{1}$ with an equivariant action of $\boldsymbol{\mu}_{r_{1}}$. Let $\tilde{Y}_{Z_{1}}$ be the normalization of $Y$ in the structure sheaf of $Y_{\text {gen }} \times_{X} Z_{1}$; it is the same as the quotient $\tilde{Y}_{Z} / \boldsymbol{\mu}_{r_{0}}$. The scheme $\tilde{Y}_{Z_{1}}$ is $\mathrm{S} 2$, but it is not necessarily flat over $Z_{1}$. The group $\boldsymbol{\mu}_{r_{1}}$ acts on $\tilde{Y}_{Z_{1}}$. We claim that the groupscheme $\mathcal{G}_{Z_{1}}^{\prime}$ acts on $\tilde{Y}_{Z_{1}}$, equivariantly under $\boldsymbol{\mu}_{r_{1}}$. Indeed the projection $\mathcal{G}_{Z_{1}}^{\prime} \times_{Z_{1}} \tilde{Y}_{Z_{1}} \rightarrow \tilde{Y}_{Z_{1}}$ is flat, and being the pullback of the flat Cohen-Macaulay scheme $\mathcal{G}_{Z_{1}}^{\prime} \rightarrow Z_{1}$ over the CohenMacaulay $\tilde{Y}_{Z_{1}}$, the scheme $\mathcal{G}_{Z_{1}}^{\prime} \times_{Z_{1}} \tilde{Y}_{Z_{1}}$ is Cohen-Macaulay as well, in particular S2. We have a birational map $\mathcal{G}_{Z_{1}}^{\prime} \times_{Z_{1}} \tilde{Y}_{Z_{1}} \rightarrow \tilde{Y}_{Z_{1}} \times_{Z_{1}} \tilde{Y}_{Z_{1}}$ which is an isomorphism in codimension 1. The closure of its graph is finite birational over $\mathcal{G}_{Z_{1}}^{\prime} \times_{Z_{1}} \tilde{Y}_{Z_{1}}$ and is an isomorphism in codimension 1 , so there is a section, therefore the map is regular. 
Denote by $\mathcal{X}=\left[Z_{1} / \boldsymbol{\mu}_{r_{1}}\right], \mathcal{Y}=\left[\tilde{Y}_{Z_{1}} / \boldsymbol{\mu}_{r_{1}}\right]$, and $\mathcal{G}^{\prime}=\left[\mathcal{G}_{Z_{1}}^{\prime} / \boldsymbol{\mu}_{r_{1}}\right]$ the Artin stacks corresponding to the flat action groupoids. We have

Theorem 3.2.1. The stack - curve $\mathcal{X}$ is nodal, with dense open representable substack $X_{\mathrm{sm}}$; the morphism $\mathcal{G}^{\prime} \rightarrow \mathcal{X}$ is a finite flat group scheme acting on the nodal $\mathcal{Y}$; and the associated morphism $\mathcal{X} \rightarrow \mathfrak{G}$ to the moduli stack of group schemes is representable.

So in view of $\aleph-\mathrm{V} 2$, one may be justified in calling $\mathcal{X} \rightarrow \mathfrak{G}$ a twisted stable map.

Question 3.2.2. Can one give examples where $Z_{1} \rightarrow X$ is not an isomorphism? Equivalently, can one give examples where $\mathcal{G}$ does not extend over the nodes as a flat group scheme over $X$ ?

Let us focus on the formal picture, in the special case $G=p$. Let $Z=\operatorname{Spec} \Lambda[[a, c]] /(a c-p)$ be the Oort-Tate versal deformation space of the group scheme $\alpha_{p}$, see [O-T]. Consider its quotient $X=Z / \boldsymbol{\mu}_{p}=\operatorname{Spec} \Lambda[[u, v]] /\left(u v-p^{p}\right)$, and denote its node by $x \in X$. The Oort-Tate universal family of group schemes $\mathcal{G}_{Z} \rightarrow Z$ descends to a group scheme

$$
\mathcal{G}^{\prime} \rightarrow X^{\prime}:=X \backslash\{x\}
$$

which does not extend over $x$, since the action of $\boldsymbol{\mu}_{p}$ on $\alpha_{p}$ is nontrivial. The question on this formal level is,

does there exist a ramified cyclic covering $Y \rightarrow X$ of degree $p$, with $Y$ a nodal curve over $\Lambda$, such that the S2-saturation $Y^{\prime}$ of the pullback $Y \times_{X} X^{\prime}$ is a principal $\mathcal{G}^{\prime}$-bundle?

Following $\aleph-\mathrm{C}-\mathrm{V}]$ we have the following:

Corollary 3.2.3. Consider the quotient stack $\tilde{\mathcal{X}}:=\left[\mathcal{Y} / \mathcal{G}^{\prime}\right]$. Then $\mathcal{Y} \rightarrow \tilde{\mathcal{X}}$ is a principal $\mathcal{G}^{\prime}$-bundle, and the associated morphism $\mathcal{X} \rightarrow \mathfrak{B}$ to the classifying stack of the universal groupscheme over $\mathfrak{G}$ is representable.

So, in view of $\aleph-\mathrm{C}-\mathrm{V}$, we might call $\mathcal{Y} \rightarrow \tilde{\mathcal{X}}$ a twisted $\mathcal{G}$-bundle.

This suggests an approach to lifting covers from characteristic $p$ to characteristic 0 , by breaking in to two stages: lifting group-schemes (over $\mathcal{X}$ or over $\tilde{\mathcal{X}}$ ), and then lifting the covers. Recent work of Wewers [W3] seems to support such an approach.

\section{REFERENCES}

$[\aleph-\mathrm{C}-\mathrm{V}] \quad$ D. Abramovich, A. Corti and A. Vistoli, Twisted bundles and admissible covers, Communications in Algebra, to appear.

[§-O2] D. Abramovich and F. Oort, Stable maps and Hurwitz schemes in mixed characteristic. Advances in algebraic geometry motivated by physics (Lowell, MA, 2000), E. Previato, ed., 89-100, Contemp. Math., 276, Amer. Math. Soc., Providence, RI, 2001

[§-V1] D. Abramovich and A. Vistoli, Complete moduli for families over semistable curves, preprint, math.AG/9811059 ICTP lecture notes series, to appear.

[§-V2] - , Compactifying the space of stable maps, J. Amer. Math. Soc. 15 (2002), no. 1, 27-75.

[Bo] I. I. Bouw, Reduction of the Hurwitz space of metacyclic covers, preprint math.AG/0204043

[B-W1] I. I. Bouw and S. Wewers, Reduction of covers and Hurwitz spaces, preprint math.AG/0005120

[B-W2] - , Stable reduction of modular curves, preprint math.AG/0210363

[D-M] P. Deligne and D. Mumford, The irreducibility of the space of curves of given genus, Inst. Hautes Études Sci. Publ. Math. No. 36 (1969), 75-109.

[He] Y. Henrio, Arbres de Hurwitz et automorphismes d'ordre $p$ des disques et des couronnes p-adiques formels, preprint math.AG/0011098

[O-T] F. Oort and J. Tate, Group schemes of prime order, Ann. Sci. École Norm. Sup. (4), 3 (1970), 1-21.

[Ra] M. Raynaud, spécialisation des revêtements en caractéristique $p>0$. Ann. Sci. École Norm. Sup. (4) 32 (1999), no. 1, 87-126. 
[Ro] M. Romagny, Sur quelques aspectes des champs de revêtements de courbes algébriques, Ph.D. Thesis, University of Grenoble, 2003.

[Sa1] M. Saidi, Torsors under finite flat group schemes of rank $p$ with Galois action, preprintdy math. AG/0106246

[Sa2] - Wild ramification and a vanishing cycle formula, preprint math.AG/0106248

[Sa3] - Galois covers of degree p: semi-stable reduction and Galois action, preprint math.AG/0106249

[W1] S. Wewers, Reduction and lifting of special metacyclic covers, preprint math.AG/0105052

[W2] - , Three point covers with bad reduction, preprint math.AG/0205026

[W3] - Formal deformation of curves with group scheme action, preprint math. AG/0212145

Department of Mathematics, Boston University, 111 Cummington Street, Boston, MA 02215, U.S.A.

Current address: Department of Mathematics, Box 1917, Brown University, Providence, RI, 02912

E-mail address: abrmovic@math.bu.edu; abrmovic@math.brown.edu 